\title{
EDITORIAL
}

\section{EL CUMPLIMIENTO DE LOS DERECHOS HUMANOS: PRIMERA MEDIDA DE SALUD PÚBLICA}

\author{
Rafael Jiménez García-Pascual. \\ Dirección General de Salud Pública de la Consejería de Sanidad y Servicios Sociales de la Comunidad Autónoma \\ de Madrid. Miembro del Equipo de profesionales de la salud de la Sección Española de Amnistía Internacional.
}

En 1998 se conmemora el cincuenta aniversario de la Declaración Universal de los Derechos Humanos ${ }^{1}$, aprobada el doce de diciembre de 1948. En 1997 también se cumplieron cincuenta años de la promulgación del Código de Nuremberg ${ }^{2}$. Es, por tanto, una buena ocasión para subrayar la relación de interdependencia existente entre ambos temas, la Salud y los derechos humanos.

Es interesante constatar que en la citada Declaración Universal se liga la salud al derecho a un nivel de vida adecuado (artículo 25) y creemos que ello es consecuencia de que, más que tener derecho exclusivamente a la salud, lo que se pretende es que todos los condicionantes de la misma estén garantizados, para que también los propios individuos sean los responsables de mantenerla. Así, en la citada Declaración Universal de los Derechos Humanos, se pone el énfasis en el derecho a la educación, a la dignidad y la libertad de la persona, a la justicia, a la seguridad, al trabajo y al ocio, así como en el derecho a la vida sin estar sometido a ningún tipo de trato cruel, inhumano o degradante, ni a torturas. Por ello, debe admitirse que la primera medida de salud pública que pueden adoptar los gobiernos es el respeto de los derechos humanos.

Por otra parte, aunque está limitado a la experiencia concreta de los ensayos realizados por los médicos nazis con los prisioneros de los campos de concentración durante la
Segunda Guerra Mundial, el Código de Nuremberg representa, igualmente, un avance sustancial en el respeto de los derechos humanos, al ligar el desarrollo científico con el derecho de los sujetos a dar el consentimiento voluntario, tanto para comenzar como para decidir finalizar su participación en una investigación en el momento que lo desee y, también, al introducir la prohibición de cualquier tipo de sufrimiento o lesión, físicas y montales, de los sujetos participantes.

En nuestro país disponemos, en principio, de un sistema de salud que nos garantiza la adecuada atención sanitaria y que nos permite mantener un determinado nivel de bienestar. Sin embargo, los principios en los que se basa este sistema, universalidad, equidad y solidaridad, pueden ser igualmente cuestionados desde la perspectiva de los derechos humanos. La universalidad se relativiza fácilmente cuando pensamos en los aspectos de la accesibilidad al sistema sanitario. La educación sanitaria, el componente de distribución geográfica, las propias creencias y actitudes de la sociedad española son aspectos que distorsionan una justa accesibilidad y que, por tanto, no permiten la universalidad del sistema. Aquí, además, hay que poner especial énfasis en la falta de acceso al sistema que sufren las personas que padecen la condición de inmigrantes, tanto legales como ilegales.

De igual forma, se pueden comentar los aspectos relacionados con la equidad. Al 
pensar en las diferencias socio-económicas 0 en las debidas a la edad y sexo de las personas, tenemos a nuestra disposición los resultados de diferentes trabajos de investigación, en los que se demuestra la existencia de diversos tipos de desigualdades, siendo un ejemplo la última publicación sobre indicadores de salud del Ministerio de Sanidad y Consumo ${ }^{3}$.

Por último, en lo relativo a la solidaridad del sistema, ya sea en el propio territorio o con terceros países, tema este actualmente muy en boga, observamos que no siempre se trabaja desde la perspectiva de los derechos humanos. Dos ejemplos concretos podrían ser tanto la solidaridad intergeneracional, es decir, la distribución de los recursos según las necesidades y otro la política sanitaria de cooperación internacional. En el primer caso, es de sobra conocida la falta de recursos sociosanitarios existente en relación a los colectivos que los necesitan. El segundo se refiere a la distribución de los recursos con respecto a otros países, que se hace en base a políticas que no tienen en cuenta la situación de los derechos humanos en los mismos.

Todo lo anterior, nos conduce a la preocupación de profundizar en los déficits de nuestro sistema sanitario $\mathrm{y}$, al mismo tiempo, a promocionar los derechos humanos de los ciudadanos para conseguir el mayor nivel de bienestar posible.

La idea de ligar la salud con los derechos humanos está en auge, ya que cs evidente que ambos están estrechamente relacionados con las preocupaciones básicas de los individuos, en especial en los aspectos referentes a la salud pública. Estamos influenciados, desde hace un par de décadas, por el aspecto multidisciplinar y globalizador del concepto de salud para la consecución de un completo estado de bienestar físico, mental y social y, si leemos los artículos de la Declaración Universal, concluímos que los derechos humanos también tratan de conseguir un mundo donde la meta principal sea el bienestar, lo más completo posible, de los ciudadanos.
En consonancia con ello, ciertos profesionales de la salud pública hablan ya de una nueva era de esta especialidad, que se estructura alrededor de los derechos humanos ${ }^{4}$. Durante la redacción y corrección de este editorial, hemos tenido la desagradable noticia del fallecimiento de uno de ellos, J. Mann en un accidente aéreo. Me gustaría aprovechar la ocasión para rendirle un homenaje y reconocer públicamente su dedicación y apoyo al tema que nos ocupa. En cualquier caso, lo anterior no deja de ser una vuelta de tuerca más sobre que el concepto de salud no es unitario, estanco, absoluto e individual, sino que es plural, influenciable, abierto y social.

Otro aspecto de la relación entre la salud y los derechos humanos que, sin embargo, nunca parece promocionarse, es el de que la salud es también un derecho individual de la persona y como tal un derecho fundamental, del que se tiene que responsabilizar para tomar sus propias decisiones y que no puede ser dejado exclusivamente en manos de profesionales y «expertos». La Administración tiene como deber inexcusable garantizar el derecho a la salud, pero debe dejar la elección al individuo, lo que además está íntimamente relacionado con la tan manida participación del ciudadano.

En relación con esta participación, queremos resaltar el importante papel desempeñado por las ONGs en la sociedad, tanto a nivel nacional como internacional, y tanto dentro del apartado sanitario como en el de los derechos humanos. En relación con la asistencia sanitaria, conocemos la gran actividad de determinadas organizaciones en momentos críticos de ayuda a personas desplazadas, perseguidas, afectadas por desastres naturales y guerras, etc. Igualmente hacen una labor indispensable en la asistencia a colectivos desprotegidos dentro de nuestro propio país. Pero, enlazando más directamente con la conmemoración del 50 aniversario de la Declaración Universal de los Derechos Humanos señalada al principio de este editorial, pensamos que la atención se debe dirigir más a tratar de 
ligar esa asistencia a los problemas de la falta de respecto a los derechos humanos y a tener presente también otros aspectos que son menos espectaculares, como la inversión constante para elevar el nivel sanitario de la población y no sólo en situaciones de tragedia y de apelación a la mala conciencia, y que influyen decisivamente en ese nivel de bicnestar que pretendemos conseguir, tanto en nuestro país como fuera de él.

En esta fecha del cincuenta aniversario de la Declaración Universal de los Derechos Humanos, no podemos ser complacientes con la situación a la que nos enfrentamos en nuestro país. No se puede dejar de resaltar el avance que han tenido tanto la medicina asistencial como la salud pública en las dos últimas décadas, pero no nos podemos dejar llevar por la autosatisfacción, ya que debemos continuar en el avance de la integración de los derechos humanos en el campo de la salud. Esta tarea se tiene que introducir en cada una de las grandes áreas, la de la prevención, la de la asistencia y la de la investigación.

Sólo mostraremos algunos ejemplos en cada una de ellas sobre las que podemos trabajar y avanzar. En cuanto a la prevención, podemos tomar los ejemplos de maltrato a niños y maltrato a las mujeres, el trabajo infantil, la existencia de poblemas culturales asociados a personas procedentes de países con prácticas denigrantes, como puede ser la mutilación genital femenina, etc. Con respecto a la asistencia, podemos hablar de los colectivos estigmatizados, como son los enfermos de sida y los consumidores de drogas intravenosas, la asistencia que se ofrece en las prisiones, el trato que se da en los centros de acogida de refugiados, los impedimentos para tener una adecuada planificación familiar, la información sobre tóxicos ambientales y laborales, etc. Por último, en lo relativo a la investigación, se debe cuidar especialmente el cumplimiento de todos los principios relacionados con la bioética, en particular, el derecho a la confidencialidad, la autonomía, la información veraz, el consentimiento informado y el evitar los riesgos debidos a manipulaciones o tratamientos.

La lista podría ser muy amplia, pero creemos que como introducción es suficiente, aunque es preciso no dejar de resaltar un aspecto que, aunque no está tan directamente relacionado con la labor diaria en nuestro país, constituye uno de los elementos clave de las organizaciones que trabajan en el campo de los derechos humanos, en especial cuando se relacionan con el trabajo sanitario: la participación del personal sanitario en los procesos de tortura, malos tratos y ejecución de la pena capital, que, desgraciadamente, no sólo no disminuye sino que se incrementa día a día, teniendo como ejemplo paradigmático la situación existente en los Estados Unidos.

Asimismo, es importante resaltar las violaciones que sufre ese mismo personal sanitario de sus derechos en aquellos países donde no se respetan o, bien, se les persigue por no colaborar en las agresiones a los derechos humanos de otras personas.

No queremos ccrrar este tema sin citar un interesante trabajo de S.R. Benatar ${ }^{5}$ recientemente publicado en el American Journal of Public Health con el que estamos plenamente de acuerdo. En él se cuestiona la realidad de la globalización económica en la que estamos inmersos, ya que al tener esa globalización como prioridades la explotación de los países en desarrollo y el militarismo, impide, al mismo tiempo, un acceso amplio a la educación, la sanidad, el empleo y otras actividades sociales que promueven la realización de los derechos humanos de miles de millones de habitantes del planeta. En concreto, señala Benatar que los modelos de mercado están fracturando los sistemas nacionales de salud y que la solución se encontraría en ligar el crecimiento económico al cumplimiento de los derechos humanos, para promover el desarrollo y bienestar, y no exclusivamente el consumismo.

Siguiendo su ejemplo, es importante y necesario que se inicic y amplíc la colaboración de personas dedicadas a la defensa de los derechos humanos en las publicaciones científi- 
cas en nuestro país, como ya viene ocurriendo desde hace tiempo en determinados países de cultura anglosajona y en las más prestigiosas revistas médicas internacionales. Por ello, es de agradecer la petición de colaboración con este editorial y la oportunidad que se brinda para su amplia difusión, deseando que éste sea el comienzo de un fructífero campo.

\section{BIBLIOGRAFÍA}

1. Declaración Universal de Derechos Humanos, A.G. Res. 217A(III), ONU Doc. A/810, pág. 71. 1948.
2. Shuster, E. Fifty Years Later: The Significance of the Nuremberg Code. NEJ Med 1997; 337(20): 1.436-40.

3. Regidor, E., Rodríguez, C., Gutiérrez-Fisac, J.L. Indicadores de Salud. Tercera Evaluación en España del Programa Regional Europeo Salud para Todos. Madrid: Ministerio de Sanidad y Consumo; 1995.

4. Mann, J. Human Rights and the New Public Health. Health Hum Rights 1995; 1(3): 229-33.

5. Benatar, S.R. Global Disparities in Health and Human Rights: A Critical Commentary. Am J Public Health, 1998; 88(2): 295-300. 